\title{
Clinical outcomes and risk stratification for papillary thyroid carcinoma presenting with distant metastasis before the era of tyrosine kinase inhibitors
}

\author{
Kenichi Matsuzu ${ }^{1)}$, Kiminori Sugino ${ }^{1)}$, Katsuhiko Masudo'), Kazunori Mori' ${ }^{1)}$, Reiko Ono ${ }^{1)}$, \\ Hiroshi Takami ${ }^{1)}$ and Koichi Ito ${ }^{1)}$ \\ 1) Department of Surgery, Ito Hospital, Tokyo 150-8308, Japan \\ 2) Department of Surgery, Yokohama City University Hospital, Kanagawa 236-0004, Japan
} Haruhiko Yamazaki ${ }^{1)}{ }^{2)}$, Chie Masaki ${ }^{1)}$, Junko Akaishi ${ }^{1)}$, Hames Kiyomi Yamada'), Chisato Tomoda'), Akifumi Suzuki ${ }^{1)}$, Takashi Uruno ${ }^{1)}$, Keiko Ohkuwa'), Wataru Kitagawa'), Mitsuji Nagahama'),

\begin{abstract}
Radioactive iodine (RAI) therapy has been the mainstay of treatment for papillary thyroid carcinoma (PTC) patients with distant metastasis (DM). Although tyrosine kinase inhibitors (TKIs) were introduced for the treatment of RAI refractory metastatic thyroid carcinoma several years ago, clinical outcomes for PTC patients with DM treated using RAI therapy remain unclear. We retrospectively examined 64 PTC patients ( 9 men, 55 women) with DM at diagnosis treated using RAI therapy without administration of any kind of chemotherapy or TKIs. Median age of patients was 58 years. Site of DM was the lungs $(n=59)$, bone $(n=3)$, and pleural dissemination $(n=2)$. No patients showed multiple-organ metastases at diagnosis. By the end of the study period, 21 patients had died of PTC. Cause-specific survival rates at 10, 15, and 20 years after initial surgery were $68.2 \%, 63.6 \%$ and $61.1 \%$, respectively. Uni- and multivariate analyses identified age $\geq 55$ years (HR $3.1, p=0.023$ ), site of DM other than the lungs (HR 13.4, $<<0.0001$ ), and DM with no RAI avidity (HR 5.1, $p=0.0098$ ) as factors independently associated with disease-related death. When analyses were restricted to patients with lung metastasis ( $n$ $=59$ ), surgical non-curability was another independent risk factor (HR 5.2, $p=0.0047$ ) in addition to age and RAI avidity. According to risk stratification analysis based on these risk factors, patients with site of DM other than the lungs or with lung metastasis showing $\geq 2$ risk factors among age $\geq 55$ years, DM with no RAI avidity, and surgical non-curability are expected to show higher mortality rates.
\end{abstract}

Key words: Papillary thyroid carcinoma, Distant metastasis, Radioactive iodine therapy, Tyrosine kinase inhibitors

PAPILLARY THYROID CARCINOMA (PTC) sometimes metastasizes to distant organs, and distant metastasis (DM) is associated with disease-related death in PTC patients $[1,2]$. For many years, radioactive iodine (RAI) has been the mainstay of therapy for metastatic thyroid carcinoma. Patients who failed to achieve control of DM with RAI therapy had no choice beyond thyrotropin (TSH)-suppression therapy until the advent of two tyrosine kinase inhibitors (TKIs), sorafenib and lenvatinib, for RAI-refractory, recurrent or metastatic thyroid carcinoma [3, 4]. However, the definition of RAI refractory disease, as the key criterion for starting TKI therapy, is challenging. Further, some patients with failure of RAI therapy can still expect long-term disease stability with-

Submitted Feb. 26, 2020; Accepted Mar. 30, 2020 as EJ20-0081 Released online in J-STAGE as advance publication Apr. 28, 2020 Correspondence to: Kenichi Matsuzu, MD, Department of Surgery, Ito Hospital, 4-3-6 Jingumae, Shibuya-ku, Tokyo 150-8308, Japan. E-mail: k-matsuzu@ito-hospital.jp out any systematic therapy $[2,5]$. Accurate understanding of the clinical outcomes and risk factors for disease death of PTC patients with DM treated with RAI therapy and TSH suppression is thus extremely important for effective use of TKIs. Ito et al. [6] reported, based on retrospective study of 71 PTC patients presenting with DM, that age $\geq 55$ years, having tumor larger than $4 \mathrm{~cm}$, and massive extrathyroidal extension were independent risk factors for disease death. Although several other reports have examined the prognosis and risk factors for disease death of patients with metastatic thyroid carcinoma, most of those studies included both cases with DM from PTC and cases with DM from follicular thyroid carcinoma (FTC) [7-11]. However, PTC and FTC would show different biological behaviors [12]. Indeed, Sampson et al. reported that the histology of FTC represents an independent risk factor for disease-related death in DTC patients presenting with DM [8]. The clinical characteristics of patients with DM from PTC thus remain unknown 
and need to be elucidated separately from those of patients with FTC to better understand which patients will gain clinical benefit from conventional TSH suppression and RAI therapies.

The present study was designed to retrospectively examine the temporal course and clinical outcomes of PTC patients presenting with DM treated with TSH suppression and RAI therapies, and to identify prognostic factors for disease-related death and possible future candidates for TKI therapy.

\section{Material and Methods}

\section{Patients}

Between 1986 and 2004, a total of 5,695 consecutive PTC patients underwent initial surgery at Ito Hospital, Japan. We reviewed the original medical records retrospectively and collected information on patient characteristics, operative findings, microscopic pathological findings, postoperative treatments, and clinical outcomes. The diagnosis of PTC was confirmed based on the World Health Organization classification system [13]. Patients with histological sections containing poorly differentiated thyroid carcinoma or undifferentiated thyroid carcinoma were excluded from the present study. Among the 5,695 PTC patients, 65 (1.1\%) had DM at diagnosis, corresponding to class M1 in the UICC TNM classification [14]. After excluding a 74-year-old woman who had uterine cancer with local recurrences while undergoing chemotherapy treatment, a total of 64 patients were enrolled as subjects in this study. Patients who did not show DM at diagnosis but developed metachronous DM postoperatively, as a recurrence, were not included in the present study. Median follow-up was 11.0 years (range, $0.3-28.6$ years). The present study was reviewed and approved by the ethics board at our institution and was conducted in accordance with the Declaration of Helsinki.

\section{Clinicopathological information (Table 1)}

Table 1 presents the clinicopathological information of patients. Median age at the time of the initial surgery was 58 years (range, 9-82 years). The majority of patients (71.9\%) showed a primary tumor $>20 \mathrm{~mm}$ in diameter, whereas 4 patients (6.3\%) had tumors $<10 \mathrm{~mm}$ in diameter. Extrathyroidal invasion of the primary tumor was determined by the surgeon based on findings from the initial surgery. Clinical lymph node metastasis was determined preoperatively by ultrasonography (US) and/or computed tomography (CT), with or without fine needle aspiration cytology. DM was preoperatively diagnosed from X-rays, CT, and/or MRI, and by histological biopsy in several cases. Our study did not include patients with DM diagnosed by postoperative ${ }^{131} \mathrm{I}$ whole-body scans without any other preoperative radiological evidence, such as X-rays or CT. The most frequent organ site of metastases was the lungs, with 59 cases $(92.2 \%)$. No patients in the present study showed metastases in two or more organs at diagnosis. Lung metastases, which were preoperatively assessed by $\mathrm{CT}$, were $<10 \mathrm{~mm}$ in diameter in most cases $(86.4 \%)$.

\section{Therapeutic information (Table 2)}

The standard initial surgery was total thyroidectomy followed by routine lymph node dissection of the central compartment (level VI) and lateral compartment (level II-V). The initial surgery was followed by subsequent administration of an ablation dose of ${ }^{131} \mathrm{I}(30 \mathrm{mCi} ; 1,110$ $\mathrm{MBq}$ ), as previously established in accordance with Japanese legal restrictions. Patients with DM showing some uptake of ${ }^{131}$ I were primarily treated by administration of a therapeutic dose of ${ }^{131} \mathrm{I}(100 \mathrm{mCi} ; 3,700 \mathrm{MBq})$. Repeated administrations were decided on an individual basis. None of the patients in this cohort were treated with chemotherapy or TKIs. Palliative external irradiation therapy was performed as required to ease the pain of bone metastasis.

Based on the above strategy, most patients were treated with total thyroidectomy, while 4 cases underwent hemithyroidectomy $(n=2)$ or subtotal thyroidectomy $(n=2)$ due to severe comorbidity and/or extreme age. Locoregional tumors were completely removed in 51 patients $(79.7 \%)$. Non-curative factors in the remaining 13 patients with incomplete removal included unresectable advanced primary tumors that were attached to the trachea and/or esophagus $(n=5)$, mediastinal lymph nodes $(n=1)$, lymph nodes in the lateral compartment that were not dissected due to severe comorbidity $(n=$ 1), and advanced primary tumors and/or metastatic bulky lymph nodes invading bilateral recurrent laryngeal nerves $(n=6)$. In cases of bilateral recurrent laryngeal nerve invasions, while the ipsilateral laryngeal nerve was sacrificed to ensure that no tumor cells would remain, the contralateral nerve was preserved even if tiny residual tumor was present, in order to avoid tracheostomy.

Fifty-six patients $(87.5 \%)$ received an ablation dose of ${ }^{131}$ I (30 mCi; 1,110 MBq) RAI therapy, while the other 8 patients $(12.5 \%)$ did not undergo the procedure due to severe comorbidity, extreme age and/or patient refusal (Table 2). Metastatic lesions in 24 patients (42.9\%) showed RAI avidity, defined as abnormal uptake of ${ }^{131} \mathrm{I}$. The remaining 32 patients (57.1\%) did not exhibit uptake of RAI. Therapeutic doses of ${ }^{131}$ I were administered in 30 patients (46.9\%). Median number of courses of therapeutic dose RAI was 2 (range, 1-7). Median cumulative dose for the total ${ }^{131} \mathrm{I}$ dose administered was $230 \mathrm{mCi}$ (8,510 MBq) (range, 130-730 mCi; 4,810-27,010 MBq). 
Table 1 Clinicopathological information

\begin{tabular}{lcc}
\hline Factor & & $n$ \\
\hline Age (years) & $<55$ & $29(45.3 \%)$ \\
\hline Sex & $\geq 55$ & $35(54.7 \%)$ \\
& male & $9(14 \%)$ \\
Size of primary thyroid tumor $(\mathrm{mm})$ & female & $55(86 \%)$ \\
& $\leq 20$ & $18(28.1 \%)$ \\
& $>20$ to $\leq 40$ & $28(43.8 \%)$ \\
Extrathyroidal invasion of primary thyroid tumor & $>40$ & $18(28.1 \%)$ \\
\hline Clinical lymph node metastasis & negative & $35(54.7 \%)$ \\
Site of distant metastasis & positive & $29(45.3 \%)$ \\
& negative & $25(39.1 \%)$ \\
Size of lung metastasis $(\mathrm{mm})$ & positive & $39(60.9 \%)$ \\
\hline
\end{tabular}

Table 2 Therapeutic information

\begin{tabular}{lcc}
\hline Factor & & $n$ \\
\hline Extent of thyroidectomy & total thyroidectomy & $60(93.8 \%)$ \\
& subtotal thyroidectomy & $2(3.1 \%)$ \\
Lymph node dissection & lobectomy & $2(3.1 \%)$ \\
& CND and MND & $45(70.3 \%)$ \\
\hline Locoregional curability & CND only & $7(10.9 \%)$ \\
& sampling & $12(18.9 \%)$ \\
\hline RAI ablation $\left(30\right.$ mCi of $\left.{ }^{131} \mathrm{I}\right)$ & curative & $51(79.7 \%)$ \\
\hline RAI avidity of metastatic lesion & non-curative & $13(20.3 \%)$ \\
\hline RAI therapy (100 mCi of $\left.{ }^{131} \mathrm{I}\right)$ & done & $56(87.5 \%)$ \\
& not done & $8(12.5 \%)$ \\
\hline
\end{tabular}

Two patients with bone metastasis underwent palliative external irradiation therapy and osteoclastic activity inhibitor administration with zoledronic acid.

\section{Statistical analysis}

All data were analyzed using JMP version 12 statistical software (SAS Institute, Cary, NC). The cumulative cause-specific survival (CSS) rate was calculated. Uni- variate analyses of clinicopathological and therapeutic factors were performed using the Kaplan-Meir method with log-rank testing. Factors selected as significant in the univariate analyses were then examined in multivariate analysis using Cox proportional hazard modeling [15, $16]$. These analyses were first performed for all patients $(n=64)$, then only for those patients with lung metastasis $(n=59)$. The level of statistical significance for 


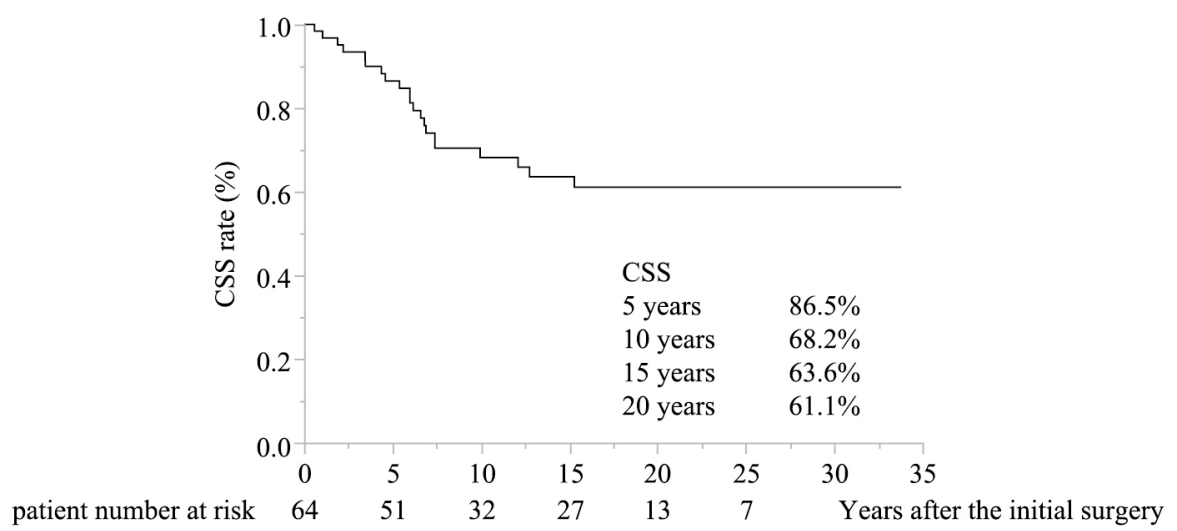

Fig. 1 Cause-specific survival (CSS) curve for papillary thyroid carcinoma patients with distant metastasis at diagnosis. Five-, 10-, 15-, and 20 -year CSS rates are $86.5 \%, 68.2 \%, 63.6 \%$, and $61.1 \%$, respectively.

results in this study was set at $p<0.05$. Factors identified as significant by both uni- and multivariate analyses are shown with hazard ratios (HRs).

\section{Results}

\section{Clinical outcomes}

A total of 21 patients had died of PTC by the end of follow-up. CSS rates at 5, 10, 15, and 20 years after initial surgery were $86.5 \%, 68.2 \%, 63.6 \%$ and $61.1 \%$, respectively (Fig. 1). The most frequent causes of death were persistent local tumors that changed to undifferentiated cancer $(n=2)$, lung metastasis $(n=12)$, bone metastasis $(n=1)$, and multiple-organ metastases $(n=6)$. With the exception of 1 case with lung metastasis who died of liver failure due to liver cirrhosis with hepatocellular carcinoma at 5.8 years after the initial surgery, none of the patients died of any other diseases during the study period.

Two patients who died primarily due to local tumors were among the 13 patients whose primary tumor was not completely removed. On the other hand, none of the 51 patients who underwent curative removal of the primary tumor died due to local tumors.

Among the 30 patients who underwent RAI therapies, 2 patients attained complete response (CR) in accordance with RECIST criteria [17]. One was a 16-year-old woman with lung metastases who attained CR after undergoing 7 courses of RAI therapy (total dose, 730 $\mathrm{mCi} ; 27,010 \mathrm{MBq}$ ), and the other was a 53-year-old man with lung metastases who achieved $\mathrm{CR}$ after 3 courses of RAI therapy (total dose, $330 \mathrm{mCi}$; 12,210 MBq). Both patients are currently alive without any evidence of recurrent or persistent disease at 21.0 and 21.5 years after the initial surgery, respectively. A total of 19 patients achieved stable disease (SD) and are still alive, whereas the remaining 9 patients, who exhibited no benefit from RAI therapy, were classified as showing progressive disease (PD) and subsequently died of PTC. Among the 34 patients who did not undergo RAI therapies, DM worsened in 13 patients, while DMs in 21 patients, all of whom showed lung metastasis alone, did not exhibit any degree of progression.

Eleven patients developed multiple-organ metastases postoperatively. These included the lungs as the site of first metastasis to bone $(n=4)$ or brain $(n=1)$ as the second, bone to lung $(n=1)$, pleural dissemination to bone $(n=2)$, lung to brain and bone as second and third metastases $(n=2)$, and bone to lung and brain $(n=1)$. At the end of follow-up, 8 of these 11 patients with multiple-organ metastases had died of PTC.

\section{Prognostic factors and stratification for disease- related deaths}

Univariate analyses showed that the risk factors significantly associated with disease-related death included age at diagnosis, site of DM, and no RAI avidity in distant metastatic lesions (Fig. 2, Table 3). Other factors examined in the present study showed no differences in CSS rates. Multivariate analysis confirmed that each of these three factors were independently associated with diseaserelated death (Table 4). DM in sites other than the lung was the strongest risk factor for disease-related death among these three. This was evidenced by the fact that all 5 patients with DM in sites other than the lung died of this disease, while 43 of the 59 patients with lung metastasis alone remained alive as of the end of the study period.

Since most patients with DM had lung metastasis alone $(92.2 \%)$, similar analyses, but omitting the site of $\mathrm{DM}$, were performed for the 59 patients with lung metastasis alone. As in analyses of all 64 patients, age at diagnosis and RAI avidity in DM represented independent risk factors for disease death. Locoregional curability of the initial surgery, which was not associated with CSS 

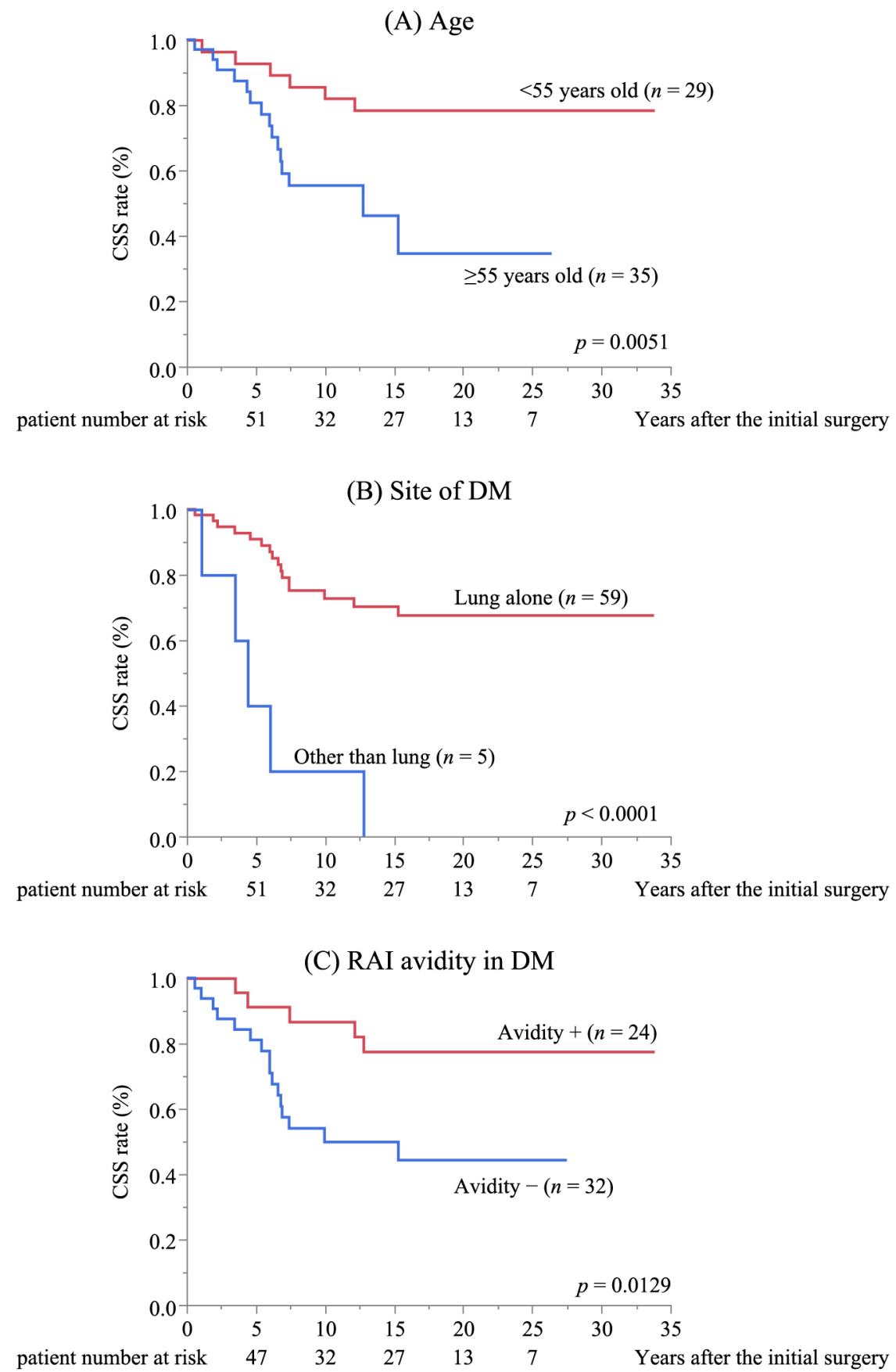

Fig. 2 Significant risk factors related to a lower cumulative survival rate are age at diagnosis $(\mathrm{A}, p=0.0051)$, site of distant metastasis (B, $p<0.0001)$, and RAI avidity in distant metastatic lesions $(\mathrm{C}, p=0.0129)$.

rates in the overall cohort of 64 patients, was identified as an independent risk factor, but other factors examined showed no differences (Table 5).

\section{Stratification of patients with DM for disease-related death}

As shown above, DM in sites other than the lungs was the strongest risk factor for disease-related death. Among patients with lung metastasis alone, we hypothesized that a greater number of risk factors would be associated with lower CSS rates. The 59 patients with lung metastasis alone were then classified simply by totaling the number of independent risks factors: 14 patients with 0 risk factors (Lung meta with Risk-0); 18 patients with 1 factor (Lung meta with Risk-1); 23 patients with 2 factors (Lung meta with Risk-2); and 4 patients with all 3 factors (Lung meta with Risk-3). CSS rates were compared among these four groups classified by number of risk factors and patients with DM in sites other than the lungs by univariate analysis using the Kaplan-Meier method 
Table 3 Univariate analysis

\begin{tabular}{|c|c|c|c|c|c|c|c|c|}
\hline \multirow{2}{*}{ Factor } & & \multirow{2}{*}{$n$} & \multirow{2}{*}{ Death } & \multicolumn{4}{|c|}{ CSS rates } & \multirow{2}{*}{$p$} \\
\hline & & & & 5-year & 10-year & 15 -year & 20-year & \\
\hline \multirow[t]{2}{*}{ Age (years) } & $<55$ & 29 & 6 & 92.9 & 82.1 & 78.6 & $\rightarrow$ & \multirow{2}{*}{0.0051} \\
\hline & $\geq 55$ & 35 & 15 & 80.7 & 55.4 & 46.2 & 34.6 & \\
\hline \multirow[t]{2}{*}{ Sex } & male & 9 & 2 & 88.9 & 76.2 & $\rightarrow$ & $\rightarrow$ & \multirow{2}{*}{0.5792} \\
\hline & female & 55 & 19 & 86.2 & 67.0 & 62.0 & 59.3 & \\
\hline \multirow[t]{2}{*}{ Size of primary tumor (mm) } & $\leq 40$ & 45 & 18 & 86.4 & 65.2 & 59.4 & 56.3 & \multirow{2}{*}{0.2628} \\
\hline & $>40$ & 19 & 3 & 86.5 & 78.7 & $\rightarrow$ & $\rightarrow$ & \\
\hline \multirow[t]{2}{*}{ Extrathyroidal invasion } & negative & 35 & 9 & 81.5 & 75.2 & 71.0 & $\rightarrow$ & \multirow{2}{*}{0.213} \\
\hline & positive & 29 & 12 & 92.9 & 58.7 & 53.4 & 48.0 & \\
\hline \multirow[t]{2}{*}{ Clinical lymph node metastasis } & negative & 24 & 9 & 81.8 & 60.6 & 53.1 & $\rightarrow$ & \multirow{2}{*}{0.3993} \\
\hline & positive & 40 & 12 & 89.2 & 72.2 & 68.9 & 65.5 & \\
\hline \multirow[t]{2}{*}{ Site of distant metastasis } & lung & 59 & 16 & 90.9 & 72.7 & 70.2 & 67.5 & \multirow{2}{*}{$<0.0001$} \\
\hline & other than lung & 5 & 5 & 40.0 & 20.0 & 0 & $\rightarrow$ & \\
\hline \multirow[t]{2}{*}{ Locoregional curability } & curative & 51 & 15 & 85.0 & 73.8 & 68.0 & 64.9 & \multirow{2}{*}{0.2572} \\
\hline & non-curative & 13 & 6 & 92.3 & 45.3 & $\rightarrow$ & $\rightarrow$ & \\
\hline \multirow[t]{2}{*}{ RAI treatment } & done & 30 & 9 & 89.8 & 76.0 & 68.7 & $\rightarrow$ & \multirow{2}{*}{0.2072} \\
\hline & not done & 34 & 12 & 83.4 & 59.0 & $\rightarrow$ & 50.6 & \\
\hline \multirow[t]{2}{*}{ RI avidity } & avid & 24 & 5 & 91.3 & 86.7 & 77.6 & $\rightarrow$ & \multirow{2}{*}{0.0129} \\
\hline & not avid & 32 & 16 & 81.0 & 49.9 & $\rightarrow$ & 44.3 & \\
\hline
\end{tabular}

Table 4 Multivariate analysis (Cox proportional ratio) in all 64 patients

\begin{tabular}{lcccc}
\hline Factor & & HR & $95 \% \mathrm{CI}$ & $p$ \\
\hline Age & $\geq 55$ years & 3.1 & $1.2-8.2$ & 0.023 \\
Site of DM & other than lung & 13.4 & $3.6-47.9$ & $<0.0001$ \\
RI avidity & no avidity & 5.1 & $1.6-20.4$ & 0.0098 \\
\hline
\end{tabular}

Table 5 Multivariate analysis (Cox proportional hazard ratio) in patients with lung metastasis alone

\begin{tabular}{lcccc}
\hline Factor & & HR & $95 \% \mathrm{CI}$ & $p$ \\
\hline Age & $\geq 55$ years & 6.8 & $1.7-27.6$ & 0.0076 \\
Locoregional curability & non-curative & 5.2 & $1.6-16.8$ & 0.0047 \\
RI avidity & no avidity & 6.6 & $1.7-43.2$ & 0.0152 \\
\hline
\end{tabular}

and log-rank test (Table 6). This analysis validated that PTC patients with DM were stratified into two risk groups: a low-risk group (Lung meta with Risk-0 and Risk-1, $n=32$ ) that showed excellent prognosis; and a high-risk group (Lung meta with Risk-2 and Risk-3, and $\mathrm{DM}$ in sites other than the lungs, $n=32$ ) that showed significantly lower CSS rates (Fig. 3).

\section{Discussion}

The present study retrospectively examined PTC patients with DM at diagnosis who were treated with TSH suppression and RAI therapies without any kind of chemotherapy or TKIs. DM in sites other than the lung was the strongest risk factor for disease-related death, a finding supported by other reports $[18,19]$. Bone was the second most common site of DM in our cohort, again supported by previous reports [20]. Lang et al. [7] analyzed one potential explanation regarding why bone metastasis was associated with poorer survival outcomes, which suggested RAI was generally less effective for bone metastasis. Wu et al. [21] recently reported that in 
Table 6 Risk stratification based on risk factors

\begin{tabular}{|c|c|c|c|c|c|c|c|}
\hline & \multirow{2}{*}{$n$} & \multirow{2}{*}{ Death } & \multicolumn{4}{|c|}{ CSS rates } & \multirow{2}{*}{ Risk stratification } \\
\hline & & & 5 years & 10 years & 15 years & 20 years & \\
\hline Lung with risk-0 & 14 & 1 & 100 & 100 & 92.3 & $\rightarrow$ & \multirow{2}{*}{ Low risk } \\
\hline-1 & 18 & 1 & 100 & 93.3 & $\rightarrow$ & $\rightarrow$ & \\
\hline-2 & 23 & 10 & 81.7 & 51.6 & 51.6 & 34.4 & \multirow{3}{*}{ High risk } \\
\hline-3 & 4 & 4 & 75.0 & 0 & & & \\
\hline Other than lung & 5 & 5 & 40.0 & 20.0 & 0 & & \\
\hline
\end{tabular}

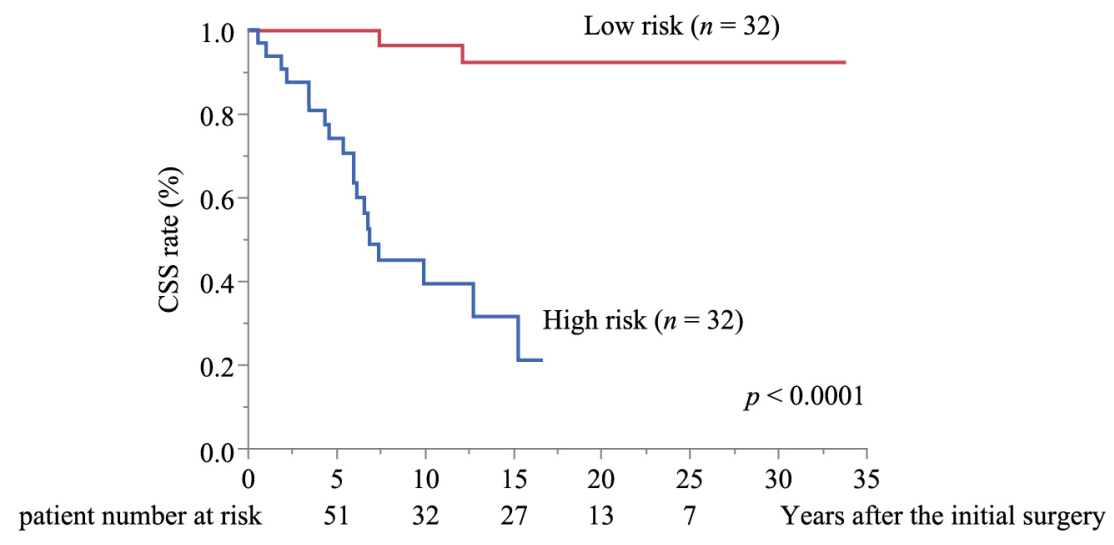

Fig. 3 Papillary thyroid carcinoma patients with distant metastasis at diagnosis were stratified into low- and high-risk groups. Five-, 10-, 15-, and 20-year CSS rates for the low-risk group were $100 \%, 96.4 \%, 92.4 \%$, and $92.4 \%$, respectively, while those for the highrisk group were $74.0 \%, 39.3 \%, 31.5 \%$, and $21.0 \%$, respectively.

DTC patients with bone metastasis, ${ }^{131} \mathrm{I}$ treatment in combination with one or more non- ${ }^{131}$ I direct and systemic treatments was associated with significantly increased overall survival compared with patients treated using ${ }^{131}$ I treatment alone. Distant metastases in multiple organs have also been reported as a risk factor for disease-related death [22], whereas our cohort did not include any patients with metastases in two or more organs at the time of diagnosis. However, our study did find that patients who developed a second DM, or second and third DMs postoperatively exhibited worse prognosis than those who did not develop second DM. Consequently, patients with DM in sites other than the lung or DMs in multiple organs at diagnosis or during follow-up are possible candidate for TKIs in the future.

Among patients with lung metastasis alone, the following three risk factors were significantly associated with disease-related death: age $\geq 55$ years; no RAI avidity in DM; and non-curative initial surgery. Among these three risk factors, age and RAI avidity in DM are wellaccepted risk factors for disease-related death in PTC patients with DM $[18,19]$. In contrast, the impact of the locoregional surgical curability of the initial surgery on the prognosis remains controversial. Sugitani et al. stated that local operative control was important even in patients with DM [18], while Ito et al. found no significant relationship between local curability and diseaserelated death [6]. The present study showed that operative curability of the locoregional tumor differed significantly in terms of CSS rates among patients with lung metastasis alone. Notably, operative curability is the only factor that can be intervened, while other independent factors (site of DM, age, and RAI avidity) are not. Locally curative operations thus should be recommended even in PTC patients with lung metastasis to reduce the number of these risk factors.

The present study had several limitations, mainly due to its retrospective design. First, the study was based on a limited number of patients from a single institution, which could imply potential for selection bias. Nevertheless, this study provided valuable information on clinical perspectives for patients with simultaneous DM from PTC, about which little has been reported due to its rarity. In addition, a randomized prospective study would not be feasible. Second, administrations of RAI therapy were determined on an individual basis, leading to variations in application times and cumulative doses of RAI therapy. In addition, a few patients who had not received RAI therapy were included. Although present study found no benefit of RAI treatment in terms of CSS rate, 
further study is required to determine the effects of repeating RAI treatments on clinical outcomes. Despite these limitations, this study is considered valuable for revealing the long-term clinical course and prognostic factors of patients with DM at diagnosis from PTC alone.

In conclusion, patients who have DM in sites other than the lungs, or patients with lung metastasis alone who have two or more of three factors (age $\geq 55$ years, no RAI avidity in lung metastasis, and locally non-curative initial operation) should not adhere to RAI therapy without any clinical benefit and should be considered as eligible candidates for TKI therapy. However, additional studies are required to ascertain whether TKI therapy might improve clinical outcomes in such cases.

\section{References}

1. Cady B, Rosai R (1988) An expanded view of risk group definition in differentiated thyroid carcinoma. Surgery 104: 947-953.

2. Hay ID, Bergstralh EJ, Goellner JR, Ebersold JR, Grant CS (1993) Predicting outcome in papillary thyroid carcinoma: development of a reliable prognostic scoring system in a cohort of 1779 patients surgically treated at one institution during 1940 through 1989. Surgery 114: 1050 1057.

3. Brose MS, Nutting CM, Jarzab B, Elisei R, Siena S, et al. (2014) Sorafenib in radioactive iodine-refractory, locally advanced or metastatic differentiated thyroid cancer: a randomised, double-blind, phase 3 trial. Lancet 384: 319 328.

4. Schlumberger M, Tahara M, Wirth LJ, Robinson B, Brose MS, et al. (2015) Lenvatinib versus placebo in radioiodine-refractory thyroid cancer. $N$ Engl J Med 372: 621-630.

5. Ringel MD (2011) Metastatic dormancy and progression in thyroid cancer: targeting cells in the metastatic frontier. Thyroid 21: 487-492.

6. Ito Y, Masuoka H, Fukushima M, Inoue H, Kihara M, et al. (2010) Prognosis and prognostic factors of patients with papillary carcinoma showing distant metastasis at surgery (M1 patients) in Japan. Endocr J 57: 523-531.

7. Lang BH, Wong KP, Cheung CY, Wan KY, Lo CY (2013) Evaluating the prognostic factors associated with cancerspecific survival of differentiated thyroid carcinoma presenting with distant metastasis. Ann Surg Oncol 20: 1329 1335.

8. Sampson E, Brierley JD, Le LW, Rotstein L, Tsang RW (2007) Clinical management and outcome of papillary and follicular (differentiated) thyroid cancer presenting with distant metastasis at diagnosis. Cancer 110: 1451-1456.

9. Haq M, Harmer C (2005) Differentiated thyroid carcinoma with distant metastases at presentation: prognostic factors and outcome. Clin Endocrinol (Oxf) 63: 87-93.

10. Schlumberger M, Tubiana M, De Vathaire F, Hill C, Gardet P, et al. (1986) Long-term results of treatment of 283 patients with lung and bone metastases from differentiated thyroid carcinoma. J Clin Endocrinol Metab 63: 960-967.

11. Mihailovic J, Stefanovic L, Malesevic M, Markoski B (2009) The importance of age over radioiodine avidity as a prognostic factor in differentiated thyroid carcinoma with distant metastases. Thyroid 19: 227-232.

12. Sugino K, Kameyama K, Nagahama M, Kitagawa W, Shibuya H, et al. (2014) Follicular thyroid carcinoma with distant metastasis: outcome and prognostic factor. Endocr J 61: 273-279.

13. DeLellis RA, Lloyd RV, Heitz PU (2004) WHO Classification of Tumours, Pathology and Genetics of Tumours of Endocrine Organs. IRAC Press, Lyon, France: 73-76.

14. Tuttle M, Haugen B, Shah J (2017) Thyroid-differentiated and anaplastic carcinoma. In: Amin MB, Edge SB, Greene F, et al (eds) AJCC Cancer Staging Manual. (8th). Springer International Publishing, New York, USA.

15. Cox DR (1972) Regression models and life tables. J R Stat Soc Series B Stat Methodol 34: 187-220.

16. Peto R, Pike MC, Armitage P, Breslow NE, Cox DR, et al. (1977) Design and analysis of randomized clinical trials requiring prolonged observation of each patients. II. Analysis and examples. Br J Cancer 35: 1-39.

17. Eisenhauer EA, Therasse P, Bogaerts J, Schwartz LH, Sargent D, et al. (2009) New response evaluation criteria in solid tumours: revised RECIST guideline (version 1.1). Eur J Cancer 45: 228-247.

18. Sugitani I, Fujimoto Y, Yamamoto N (2008) Papillary thyroid carcinoma with distant metastases: survival predictors and the importance of local control. Surgery 143: 35-42.

19. Dinneen SF, Valimaki MJ, Bergstralh EJ, Goellner JR, Gorman CA, et al. (1995) Distant metastases in papillary thyroid carcinoma: 100 cases observed at one institution during 5 decades. J Clin Endocrinol Metab 80: 2041-2045.

20. Shoup M, Stojadinovic A, Nissan A, Ghossein RA, Freedman S, et al. (2003) Prognostic indicators of outcomes in patients with distant metastases from differentiated thyroid carcinoma. J Am Coll Surg 197: 191-197.

21. Wu D, Gomes Lima CJ, Moreau SL, Kulkarni K, Zeymo A, et al. (2019) Improved survival after multimodal approach with 131I treatment in patients with bone metastases secondary to differentiated thyroid cancer. Thyroid 29: 971-978.

22. Casara D, Rubello D, Saladini G, Masarotto G, Favero A, et al. (1993) Different features of pulmonary metastases in differentiated thyroid cancer: natural history and multivariate statistical analysis of prognostic variables. $\mathrm{J} \mathrm{Nucl}$ Med 34: 1626-1631. 